\title{
Southern California Seismic Network: Caltech/ USGS Element of TriNet 1997-2001
}

\section{Egill Hauksson, Patrick Small, Katrin Hafner, Robert Busby, Robert Clayton, James Goltz, Tom Heaton, Kate Hutton, Hiroo Kanamori, Jascha Polet}

Seismological Laboratory, California Institute of Technology

\section{Doug Given, Lucile M. Jones, and David Wald}

\author{
U.S. Geological Survey, Pasadena, California
}

\section{INTRODUCTION}

The California Institute of Technology (Caltech), the United States Geological Survey (USGS), and the California Department of Conservation, Division of Mines and Geology (CDMG) are completing the implementation of TriNet, a modern seismic information system for southern California. TriNet consists of two elements, the Caltech-USGS element and the CDMG element (Mori et al., 1998). The CaltechUSGS element (Caltech-USGS TriNet) concentrates on rapid notification and archiving of data for seismological applications, while the CDMG element is focused on the needs of engineering users (Hauksson et al., 2002). All three TriNet agencies are working toward facilitating emergency response and long-term mitigation of earthquake hazards in cooperation with other agencies. The technical development of Caltech-USGS TriNet is sufficiently different from the CDMG element of TriNet to warrant a separate description.

This paper provides a technical overview of the design principles of Caltech-USGS TriNet. These principles were based on a document that stated the scientific requirements of TriNet (Jones et al., 1997). We also describe the implementation of these principles using modern technology. The implementation consisted of station deployments, establishing communications links, and developing and implementing new hardware and software for data processing and information distribution. Thus, the Caltech-USGS TriNet is an integrated project extending across many disciplines, using basic ground-motion data and seismological algorithms to generate in near real-time a sophisticated earthquake knowledgebase following earthquakes in southern California.

Caltech-USGS TriNet applies advanced technology to record both small and large earthquakes on scale. The latest generation of broadband and strong-motion sensors with 24bit digitizers is used to acquire high-fidelity ground-motion data. Real-time communication is a requirement to facilitate rapid processing and notification about seismicity for emergency management. The data acquisition systems are designed to ensure redundancy and automated processing of data. To accomplish automation, high-speed computers and advanced software form the inner workings of the CaltechUSGS TriNet system. Adopting the commercial database Oracle is an important foundation of our data management system. The automated flow of data into an accessible data center and the automatic population of the database is part of our new seismic network design and is an essential feature of Caltech-USGS TriNet. The TriNet real-time systems and database have been operating online for more than two years, processing real-time data currently from more than 375 stations, or more than 1,200 high sample-rate data channels. Many of these capabilities were tested in the $1999 M_{w} 7.1$ Hector Mine earthquake. New postprocessing and cataloggeneration approaches have also been implemented in 2001 .

Caltech-USGS TriNet is one of the first U.S. regional seismic networks that uses digital technology on a scale of 200 or more stations, with both broadband and strongmotion sensors. In comparison, the IRIS Global Seismic Network consists of 108 stations, with plans for a total of 150 stations (Hutt and Bolton, 1999). Previous digital networks, such as TERRAscope (Kanamori et al., 1997) and the Berkeley Digital Seismic Network (BDSN) (Gee et al., 1996), have been smaller than TriNet, with about 20 stations each. TriNet also benefits from the experience of other seismic networks around the world. The K-Net in Japan is another example of large-scale deployment of a digital network, although it is focused on strong motions (Kinoshita, 1998). Extensive developments of strong-motion networks in Taiwan and associated near-real-time processing of data employ somewhat different technology but have similar goals for information products following large earthquakes (Teng et al., 1997). 


\section{CALTECH-USGS TRINET MISSION AND GOALS}

The mission of the Caltech-USGS element of TriNet is to provide in a timely manner the best possible earthquake data, information, and research so as to reduce the earthquake risk in southern California. Because earthquakes cannot be predicted or prevented, the goals of TriNet reflect both shortand long-term responses:

- Operate a hardened seismographic network to record earthquake ground motions in southern California. The network must be dense enough to document the true distribution of ground motions and must be robust enough not to fail during a M 8 earthquake.

- Cooperate with other agencies working to mitigate the earthquake hazard in southern California through the recording, analysis, and distribution of information, especially the Office of Emergency Services, the Federal Emergency Management Agency, the Division of Mines and Geology, and the Southern California Earthquake Center.

- Create an easily accessible database of earthquake information in southern California for research in seismology and earthquake engineering. The earthquake catalog that lists what earthquakes have occurred can also be used to evaluate the future rate of seismicity. The database of earthquake phases will provide insight into the structure of the Earth. The high-fidelity records of the ground shaking during earthquakes will help elucidate the earthquake source, and document what level of shaking earthquakes produce and what levels buildings, both damaged and undamaged, endured, providing the knowledge society needs to build a resilient infrastructure.

- Distribute locations and magnitudes rapidly to critical users after earthquakes to facilitate decision making.

- Distribute ground shaking information rapidly after damaging earthquakes to facilitate such mitigating actions as search and rescue, fire prevention, and deployment of engineers and inspectors for building inspection, and thus save lives and property.

- Begin development of a prototype early warning system and begin collection and analysis of relevant social science data to facilitate future implementation. However, to accomplish successful implementation, funds will be needed for future SCSN/TriNet enhancements, including sufficient station density to enable rapid detection and to make it possible for SCSN/TriNet to signal that an earthquake has begun before damaging shaking arrives at more distant sites.

The goals above can be accomplished only by using modern technology for rapid recording and distribution of ground shaking information, which can serve many purposes in earthquake planning, research, response, and prototype early warning. TriNet is intended to meet all these goals in one system.

\section{REMOTE SEISMIC STATIONS}

Initially, the Southern California Seismic Network (SCSN) was mostly a short-period network installed in the early 1970 's and consisted of about 250 stations through much of the 1980's and 1990's. In the late 1980's and early 1990's, the short-period stations were supplemented by TERRAscope, a network of digital stations with both broadband and strongmotion sensors (Kanamori et al., 1991). By late 1996, the TERRAscope network had grown to about 28 stations. These stations have been upgraded and incorporated into TriNet.

Since 1997 the number of short-period stations has decreased to approximately 140 stations. These remaining remote short-period stations are needed to maintain a detection threshold of M 1.8. The most significant change in how the short-period stations are operated is the introduction of remote Earthworm hubs (Johnson et al., 1995). Today, three remote Earthworm hubs are digitizing signals locally and transmitting the data via the Internet or frame relay to Caltech-USGS in Pasadena.

When completed, Caltech-USGS TriNet will consist of 155 stations with broadband and strong-motion sensors (Figure 1). In addition, Caltech-USGS TriNet will record realtime signals from about 140 short-period stations, 12 broadband stations in the Anza Seismic Network (Vernon, 1989), and 55 strong-motion instruments operated by Caltech, USGS, USGS/NSMP, and CDMG. The total number of high data-rate channels ( 80 sps or $100 \mathrm{sps}$ ) will be in excess of 1,200 . In addition, more than 2,000 channels of low sampling rates (20 sps, $1 \mathrm{sps}, 0.1 \mathrm{sps}$ ) and state of health channels will be recorded.

\section{New Station Siting and Installation}

The station siting criteria were developed from the goals of TriNet. To monitor seismicity to a minimum magnitude of

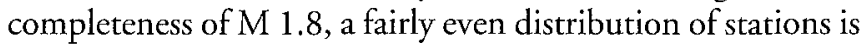
needed across southern California. Accomplishing an even geographical distribution, however, is challenging in mountainous areas and in densely populated urban areas. To facilitate the understanding of ground motions where people live, we have installed densely spaced stations within the urban areas. We have deployed stations in a variety of settings to capture both high-quality data at bedrock sites and data from sites with potentially unusual site effects.

Traditionally, strong-motion stations have been either free-field or in buildings. Following the $1994 M_{w} 6.7$ Northridge earthquake the concept of a reference strongmotion station was developed (CSMIP, written communication, 1999), in part because almost no records were available at sites close to damaged steel structures (Krawinkler et al., 1995). The idea is to record data close enough to a cluster of large buildings or structures so that the records can be correlated with the observed damage. Reference stations should be located on representative geological materials, within one mile of a business district or a group of engineered structures. These data from the immediate vicinity of earthquake-dam- 


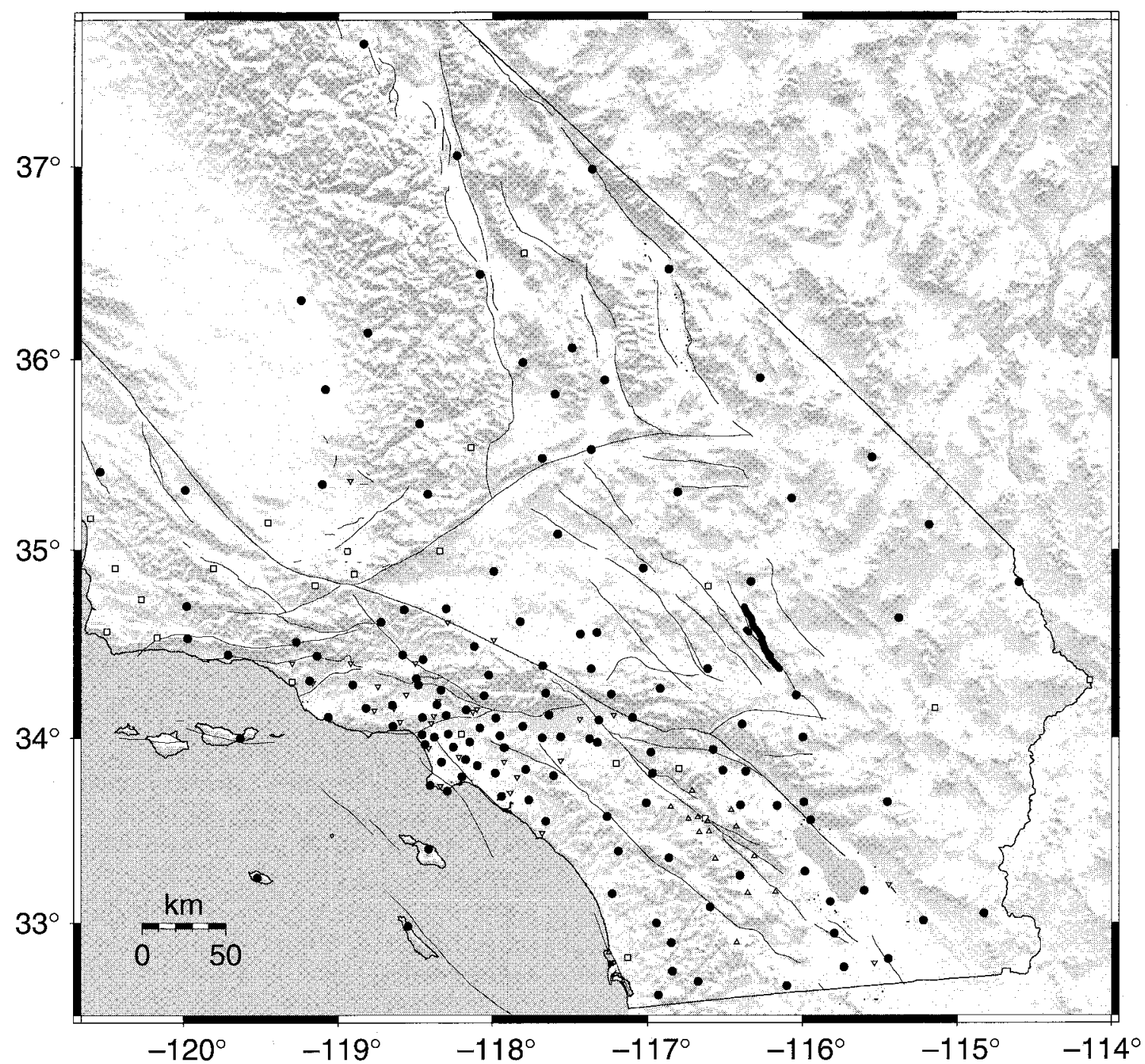

A Figure 1. Caltech-USGS TriNet stations with broadband and/or strong-motion sensors. These stations use IP communications to transmit data to the central site. Included are 135 existing stations broadband and strong-motion (solid circles) and 20 stations under construction (open squares). Stations with strong motion sensors only are shown as inverted triangles. Stations in the Anza seismic network are shown as open triangles. Surface rupture of the $M_{W} 7.1$ 1999 Hector Mine earthquake is also shown.

aged structures are important for understanding the relationship between strong ground motion and damage to structures, and thus can be used to develop improved building codes.

The broadband and strong-motion station installations are generally of two types, depending on the available facilities. The first type has sensors outside in a shallow vault at a depth of 1 to $2 \mathrm{~m}$. The datalogger and other equipment are located inside an adjacent small building. The second type of installation has the sensors, dataloggers, and other equipment all installed in a vault that is $2 \mathrm{~m}$ deep and $1.3 \mathrm{~m}$ in diameter. Installations require that sensors be at least one building dimension length away from a significant building and that they avoid repetitive noise sources such as traffic, pumps, or severe radio noise that may cause interference. Stations with only strong-motion sensors may be installed either inside a small building less than $500 \mathrm{~m}^{2}$ or in a small hut adjacent to a facility with real-time communications and AC power, or they may be connected to accelerometers installed in a shat low borehole.

\section{Sensors and Dataloggers}

The sensors used by Caltech-USGS TriNet are both broadband seismometers and strong-motion accelerometers. The broadband seismometers include a range of sensors, from very broadband (360 s to $0.1 \mathrm{~s}$ ) Streckeisen STS1 and broadband (120s to $0.02 \mathrm{~s}$ ) Streckeisen STS2, Guralp 3ESP, and $3 \mathrm{~T}$, to $(30 \mathrm{~s}$ to $0.02 \mathrm{~s})$ Guralp $40 \mathrm{~T}$ seismometers. The number of units deployed in the field and the nominal frequency response are shown in Table 1 and plotted in Figure 2, respectively. At sites with low to moderate background noise, we have deployed the higher quality sensors, and at high-noise sites we have deployed the Guralp 40T sensors. The older generation force-balance accelerometers (FBA-23) are used at 


\begin{tabular}{|lcccccc|}
\hline Manufacturer & Type & $\begin{array}{c}\text { Frequency Range } \\
\mathbf{( H z )}\end{array}$ & Sensitivity & Clip Level at $\mathbf{1 ~ ( H z )}$ & $\begin{array}{c}\text { \# of } \\
\text { seismometers } \\
\text { in \% }\end{array}$ & \# of FBA sensors in \% \\
\hline Streckeisen & STS1 & $0.0027-10$ & $2,500(\mathrm{~V} / \mathrm{m} / \mathrm{s})$ & $\sim 1(\mathrm{~cm} / \mathrm{s})$ & 5 & - \\
Streckeisen & STS2 & $0.0083-50$ & $1,500(\mathrm{~V} / \mathrm{m} / \mathrm{s})$ & $\sim 1(\mathrm{~cm} / \mathrm{s})$ & 45 & - \\
Guralp & SOT & $0.0330-50$ & $800(\mathrm{~V} / \mathrm{m} / \mathrm{s})$ & $\sim 1(\mathrm{~cm} / \mathrm{s})$ & 28 & - \\
Guralp & 3 ESP & $0.0083-50$ & $2,000(\mathrm{~V} / \mathrm{m} / \mathrm{s})$ & $\sim 1(\mathrm{~cm} / \mathrm{s})$ & 8 & - \\
Guralp & $3 T$ & $0.0083-50$ & $1,500(\mathrm{~V} / \mathrm{m} / \mathrm{s})$ & $\sim 1(\mathrm{~cm} / \mathrm{s})$ & 14 & 45 \\
Kinemetrics & FBA-23 & DC-50 & $2 \mathrm{~V} / \mathrm{g}$ & $2 \mathrm{c}\left(\mathrm{cm} / \mathrm{s}^{2}\right)$ & - & 55 \\
Kinemetrics & Epi-Sensor & DC-180 & $10 \mathrm{~V} / \mathrm{g}$ & $2 \mathrm{~g}\left(\mathrm{~cm} / \mathrm{s}^{2}\right)$ & - & - \\
\hline
\end{tabular}

a. Applies to horizontal components only.

(A)

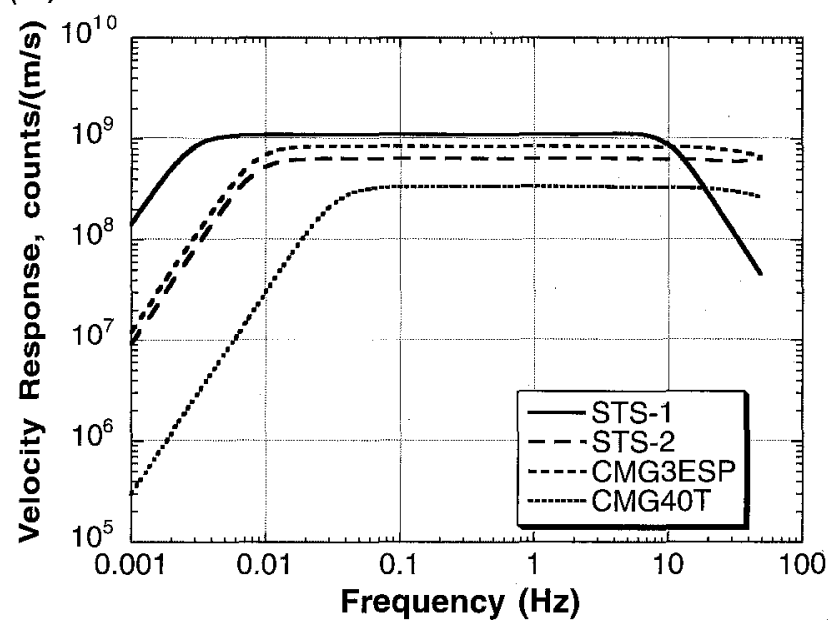

(B)

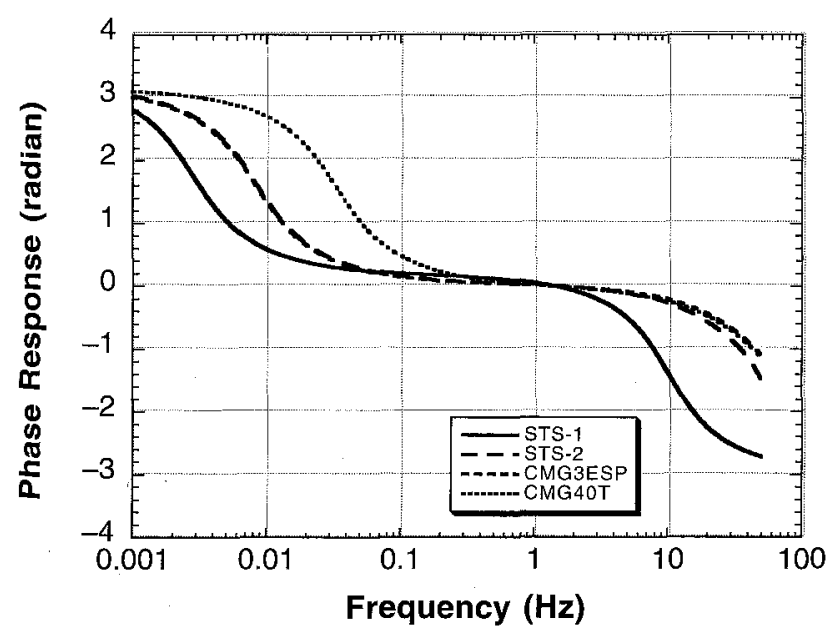

14

4 Figure 2. (A) Velocity response and (B) Phase response of the broadband seismometers (STS-1, STS-2, CMG40T, and CMG3ESP) used by Caltech-USGS TriNet. the stations deployed during the first two years of TriNet development. The more recent stations use the Episensor, a new strong-motion sensor made by Kinemetrics, Inc. that has a lower background noise level and a corner frequency of $180 \mathrm{~Hz}$.

The background noise varies from site to site. To provide weight and to minimize thermal noise we insulate the broadband sensor with a 3 " thick layer of sand. In Figure 3 we show samples of the power spectral density of the broadband STS2 seismometer data from a hard rock site (PLM) and a firm sediment site (USC). The range of background noise observed at these two sites is typical for southern California. At high frequencies $(>1 \mathrm{~Hz})$, the noise level is considerably higher at USC because of the high cultural noise level in the city. At all sites, both strong-motion and broadband sensors are anchored to prevent movement during strong shaking.

Caltech-USGS TriNet records data from eight stations with downhole sensors, each at a depth of about $100 \mathrm{~m}$. All of these stations have a surface strong-motion sensor and in some cases a surface broadband sensor. These stations are TriNet-affiliated stations that are operated in cooperation with other agencies, such as the Southern California Earthquake Center (SCEC) and the University of California at Santa Barbara (UCSB), Riverside (UCR), and San Diego (UCSD).

Caltech-USGS TriNet records about 135 stations (soon to be 155) with both broadband and strong-motion sensors, using Quanterra dataloggers of various types as shown in Table 2. The older versions of the dataloggers, Q980's and Q680's, have only acausal filter settings. The more recent dataloggers, Q4128's and Q730's, also have causal filter settings that we use for the high sampling rate data streams. The more flexible filter settings allow the use of causal filters for the high data-rate streams and acausal filters for the low datarate streams. Some of the features of the Quanterra dataloggers that we use are 24-bit resolution digitizers, on-site data storage, always-on IP telemetry capability, GPS time tagging, and flexibility in configuring the system. The GPS time-tag- 
Power Spectral Density

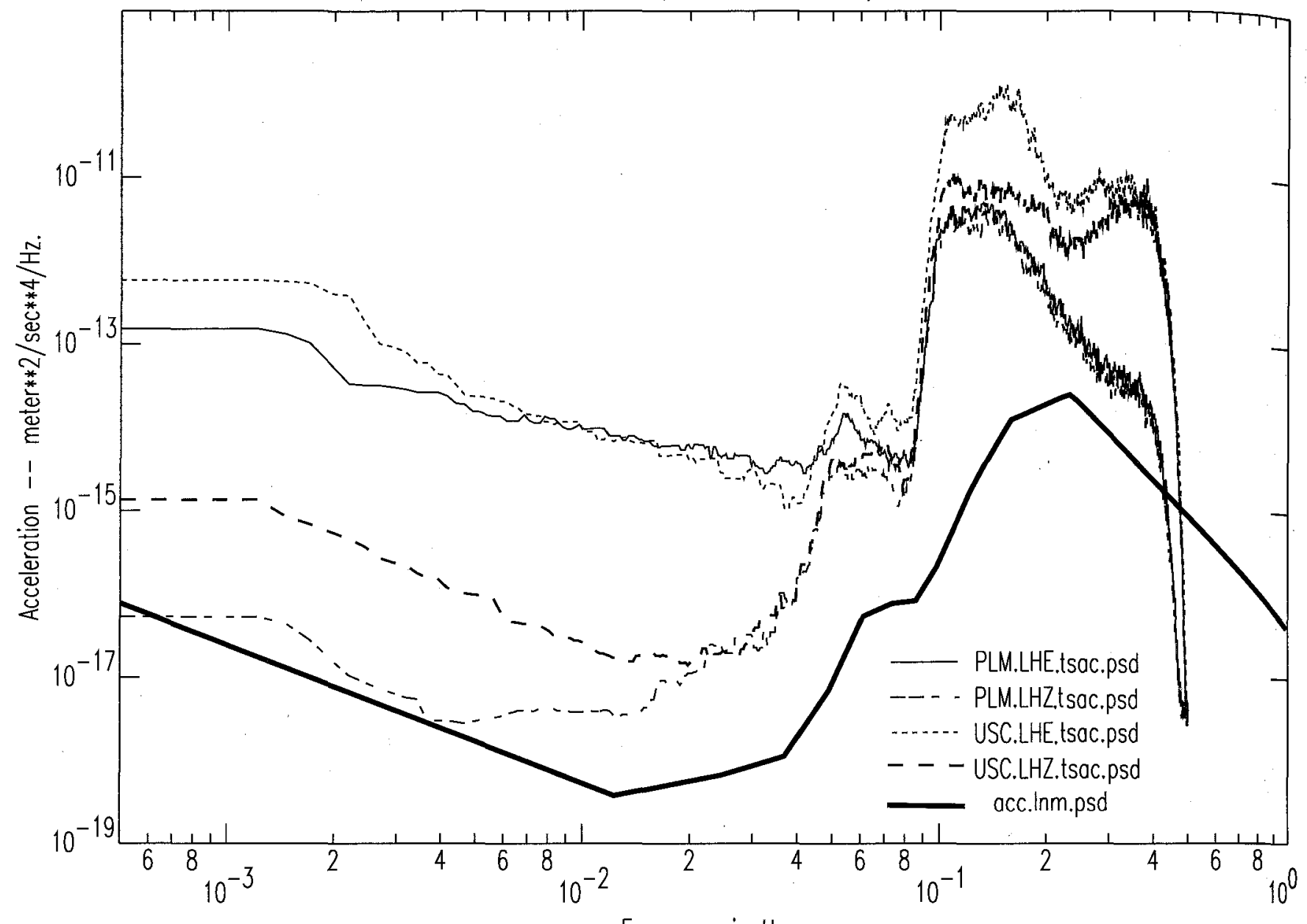

Frequency in $\mathrm{Hz}$.

$\Delta$ Figure 3. Power spectral density of background seismic noise at Palomar (PLM) and University of Southern California in the city of Los Angeles (USC) as recorded on the east and vertical components. The bottom curve is the USGS low-noise model (acc.Inm.psd).

\begin{tabular}{|lcccccccc|}
\hline \multicolumn{7}{|c|}{ Caltech-USGS TriNet: Dataloggers Transmitting Real-time Seismic Data } \\
\hline Manufacturer & Type & $\begin{array}{c}\text { On-site } \\
\text { Recording }\end{array}$ & $\begin{array}{c}\text { Communication } \\
\text { Port }\end{array}$ & $\begin{array}{c}\text { \# of } \\
\text { Channels }\end{array}$ & $\begin{array}{c}\text { Max. } \\
\text { (sps) }\end{array}$ & Causal Filter & $\boldsymbol{\mu}$ V/Counts & \# of units in \% \\
\hline Quanterra & Q980 & $1.6 \mathrm{~Gb}$ & Ethernet & 9 & 80 & $n$ & 2.38 & 7 \\
Quanterra & Q380 & $1.6 \mathrm{~Gb}$ & Ethernet & 3 & 80 & $n$ & 2.38 & 5 \\
Quanterra & Q680 & $1.6 \mathrm{~Gb}$ & Ethernet & 6 & 80 & $n$ & 2.38 & 3 \\
Quanterra & $\mathbf{Q} 4128$ & $1.6 \mathrm{~Gb}$ & Ethernet & 8 & 100 & $y$ & 2.38 & 47 \\
Quanterra & Q736 & $1.6 \mathrm{Mb}$ & SLIP & 6 & 100 & $y$ & 1.90 & 17 \\
Quanterra & $\mathrm{Q} 736 \mathrm{E}$ & $280 \mathrm{Mb}$ & Ethernet & 6 & 100 & $y$ & 1.90 & 12 \\
Kinemetrics & $\mathrm{K} 2$ & $64 \mathrm{Mb}$ & Serial & 4 & 100 & $y$ & & 9 \\
\hline
\end{tabular}


ging accuracy is better than $0.1 \mathrm{~ms}$. We also use data from status channels that monitor sensor mass position, power status, and local temperature. The availability of several communication ports and the ability to direct data telemetry to more than one receiving computer add to the resiliency of the network. The availability of state of health information helps in diagnosing the performance of telemetry, clock, and other features. Several features can be controlled remotely, including centering the seismometer mass, application of calibration signals, and resets of the GPS engines.

A master Excel file that contains all the relevant stationspecific information, such as channels to be recorded and IP address assignments for each site, is kept on a Unix server. A shell script is run to generate a set of key files to configure , each datalogger. The key files are sent using FTP to the datalogger, and once the reboot command is issued, the datalogger is ready for field deployment. TriNet has also taken advantage of the capability of remotely updating the software on the Quanterra dataloggers, implementing both complete software upgrades and minor software patches. Files are transmitted using FTP utilities to the datalogger and unpacked locally. A reboot command is then sent to the datalogger to install the new version of software. In general, the remote downloading and reboots are successful and save the expense of a visit needed for a manual reboot. The dataloggers also run a HTTP server for manual interrogation and data retrieval.

At present, Caltech-USGS TriNet records real-time data from 30 stations with only strong-motion sensors, and in the near future we plan to add another 25 strong-motion stations. These additional 25 stations are operated by CDMG TriNet and will be connected using a FRAD that will multicast the data packets to two different locations. These stations, which are K2 family instruments, each have a built-in sensor package and a flash memory card for on-site data storage. The K2 has a serial communications port for real-time packet transmission, which can also be used to control and upgrade software remotely.

Standby power for at least 72 hours is provided at each site. The equipment consists of a charger that both powers the field equipment and charges a battery. At each site we use a custom break-out box, with isolated DC converters and a low-voltage cut-off, to connect sensors, datalogger, and communication equipment to the power source.

\section{Data Communications}

Caltech-USGS TriNet uses a diverse set of data communications provided by commercial service providers to avoid single points of failure. Commercial providers also minimize our workload involved in responding to each and every outage. There is a tremendous cost advantage in having providers such as Pacific Bell perform the initial truck-roll, leaving our technical staff to address seismic-related problems.

To transmit all waveform data in real time, the CaltechUSGS TriNet follows a central site communications model that requires two-way end-to-end communications between each remote site and the central site. The digital data transmission technologies used by the Caltech-USGS element of TriNet are listed in Table 3, and a representative diagram is shown in Figure 4. The goal is to have a diverse set of communications in order to avoid losing the whole network in the case of failure of one or more links. The primary method of data transmission is frame relay provided by the phone companies, Pacific Bell (SBC) and Verizon. Other data-transmission methods include microwave links and, in one case, a spread-spectrum radio link to Caltech.

At each site with frame relay telemetry, we use a Motorola Vanguard Series Frame-Relay Assembler and Disassembler (FRAD) interface unit. At sites where only serial communications are available, an RS-232 connection is made directly to the datalogger from a spread-spectrum radio, an analog microwave modem, or a local terminal server. At several hub sites, we receive four or six remote stations via spread spectrum or a local microwave channel. The data that arrive from the remote sites are combined through a terminal server before they are transmitted via frame relay. At these sites the frame relay lines are configured to transmit at a peak data rate of $56 \mathrm{kbps}$.

We use IP communications with UDP/IP packet protocol, over either an Ethernet or a serial port. If the datalogger has an Ethernet interface and a wide-area network connection or if frame relay is available, we use the Ethernet port. If only a serial connection is available, we use a SLIP-framed UDP/IP to communicate between the datalogger and the central site. We have tested TCP/IP on some of our links. TCP/IP is not preferred because it requires more bandwidth for overhead and because restoring the link after failure is more complicated to accomplish automatically.

The digital data transmission links are specified to handle a steady data flow of about $11 \mathrm{kbps}$, with additional capacity of $8 \mathrm{kbps}$ to provide for higher data rates during major events or to recover from temporary outages of the communications circuits. This overhead is also used for diagnostic login sessions or file transfers. The approximate total data rate for Caltech-USGS TriNet will be 3-5 Mbps. The Quanterra dataloggers allow flooding the communications link with dummy packets to determine the maximum capacity of the link. This feature makes it possible to diagnose potential malfunction and to identify narrow bandwidth segments in a multiple-segment communications link.

To transmit the data from a remote station to a major communications backbone, we use several means. In some cases, the spread-spectrum radios transmit data the last several miles to the station. We have more than 35 radio links already in operation. At other sites where the "last mile" is 60-600 m, we use optical fiber connections. If the equipment is within $60 \mathrm{~m}$ of a communications port, we use category five Ethernet cables.

Several stations use the Internet to transmit data to Caltech. Three stations located at UC Santa Barbara and UC Riverside transmit data over the Internet to Caltech and UC San Diego simultaneously. We are also testing one digital sub- 


\begin{tabular}{|c|c|c|c|c|c|}
\hline Provider & Type & $\begin{array}{c}\text { CIR }^{\mathrm{a}} \\
\text { (kbps) }\end{array}$ & Communication & \# of Stations ${ }^{b}$ & Comments \\
\hline Pacific Bell & Frame relay & 19 & $\begin{array}{l}\text { Ethernet/ } \\
\text { SLIP/serial }\end{array}$ & 86 & \\
\hline Pac Bell hub sites & Frame relay & 56 & SLIP & 4 & \\
\hline Verizon & Frame relay & 19 & Ethernet/SLIP/serial & $18+(2)$ & \\
\hline \multirow[t]{2}{*}{ Verizon hub sites } & Frame relay & 56 & SLIP & 5 & \\
\hline & Spread spectrum radio & 36 & SLIP & 1 & Mt. Wilson station \\
\hline \multirow[t]{2}{*}{ Pacific Bell } & DSL/Internet & & Ethernet & 1 & PASA test station \\
\hline & Internet & & Ethernet & 10 & \\
\hline USGS & Analog microwave & 10 & SLIP & 5 & \\
\hline Southern Calif. Edison & WAN: fiber and microwave & 56 & Ethernet & 30 & $\begin{array}{l}\text { With plans to develop } \\
\text { failover to frame relay }\end{array}$ \\
\hline City of Los Angeles DWP & Wide-area network microwave & 56 & Ethernet & 6 & $\begin{array}{l}\text { Uses a microwave link to } \\
\text { Mt. Lukens }\end{array}$ \\
\hline $\begin{array}{l}\text { Coachella Valley Water } \\
\text { Distr. }\end{array}$ & Analog microwave & 19 & SLIP & 6 & $\begin{array}{l}\text { Use frame relay from the } \\
\text { hub site }\end{array}$ \\
\hline Southern Calif. Gas Co. & Microwave channels & 19 & Ethernet/SLIP & $4+(5)$ & $\begin{array}{l}\text { Will use microwave link } \\
\text { into Caltech }\end{array}$ \\
\hline San Diego Gas \& Electric & Microwave channels & 19 & Ethernet/SLIP & (1) & $\begin{array}{l}\text { Connects to So Cal Gas } \\
\text { microwave }\end{array}$ \\
\hline
\end{tabular}

a. CIR: Committed information rate or bandwidth available for a station.

b. Numbers in parentheses are additional units to be deployed

scriber line (DSL) as a way of getting data onto the Internet. Channels on microwave systems operated by the USGS, the Southern California Gas Company, and the Coachella Water District are used to transmit data from stations either to hub sites or directly to Caltech. Two different wide-area IP networks operated by the utilities (Southern California Edison and Department of Water and Power with the City of Los Angeles) are being used to send data directly to Caltech. We have installed a microwave link between a nearby transmission tower and Caltech to transmit these data independent of the phone company. A similar link connecting TriNet to the Southern California Gas Company microwave system is in progress.

All Caltech-USGS TriNet stations can switch data telemetry between two receiving computers. This is controlled from the central site with no station modifications required and is performed for scheduled maintenance on a central receiving computer or in the event of a system failure.

Further, approximately $70 \%$ of the remote stations have communications channels with sufficient bandwidth to transmit full data streams to two central sites. The 52 stations that transmit dual data streams are referred to as high-bandwidth stations. It is the goal of Caltech-USGS TriNet to have as many high-bandwidth stations as possible. At present the acquisition computers for both central sites are located in the same room at Caltech, although in the future we plan to move one of the acquisition computers to a different building. The second recording site affords protection from central malfunction of computers and other related facilities.

\section{Station Verification}

As new TriNet stations are brought on line, the data are passed through several verification steps. First, the level of background noise is checked in the data recorded over an eight-hour period during the night. Noise events of short duration, or "pings", and long-duration background noise are detected by calculating power spectra density of 8-hour-long segments of data (Figure 3). The seismometer data for a large regional event are compared to the data from the strongmotion sensors, and teleseisms are compared from separate stations to check station polarities and gains. Tilting the sensors on their sides for a few minutes tests absolute scaling and polarity of the accelerometers. We check the GPS time tago ging of the data by analyzing the state of health information recorded by the datalogger. Furthermore, in a few cases when a station is included in the routine processing, potential callibration problems may be identified, although we attempt to clear up such problems beforehand.

Station information is stored in TriNet's Oracle database This database contains both basic station information, such 


\section{Overview of TriNet Communications}

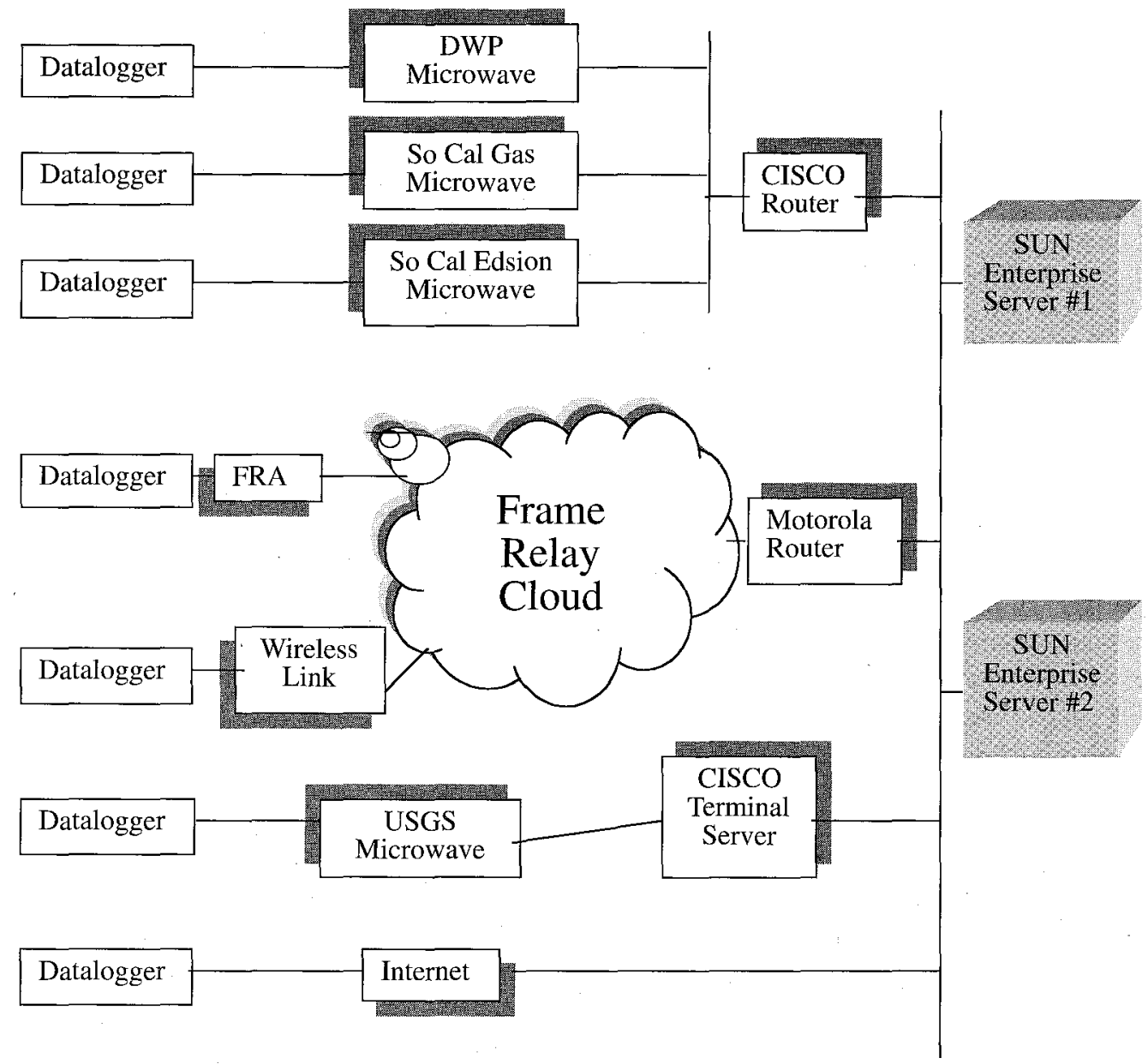

\ Figure 4. A schematic flow chart showing the major components of the TriNet communications system. Remote stations, typical communications paths, and some of the central site equipment are shown.

$i$ as locations, and the more detailed station response informa- tion. The station database schemas are the same as used by the Berkeley Digital Seismic Network (BDSN) in northern California.

\section{Network State of Health Monitoring}

To identify potential problems with the field equipment, telemetry, and time tagging of data, we have designed software to evaluate the state of health of the network. We outsourced the development of a software module (TriNetwatch) that is a three-tiered distributed system with agents, server, and frontend client. Agents collect data, control the acquisition software, and communicate over the network to the server. The server is the centralized decision-making tool that receives the agent's messages and passes on filtered data to the client.

The operator interface for TriNetwatch is a standard Web browser. TriNetwatch displays the performance and usage information for all stations. The performance information helps the operator decide what corrective actions are needed and how urgent they may be depending on how many sta- tions may have failed. The usage information is used to track stations that come on line after installation or major repair and as they move through several stages of usage. These usage stages are intended to allow proper testing of the station performance and data calibration before the station becomes a trusted real-time station.

\section{REAL-TIME DATA ACQUISITION SYSTEMS}

At the central site, Caltech and USGS in Pasadena, the time series data from the stations are acquired, processed; and archived (Figure 5). In the data acquisition phase, the data are temporarily stored in memory and quickly written to disk. The initial processing is automated and consists of deriving parametric values from the time series data to identify and quantify earthquakes. As part of the second stage of the data processing, data analysts review the content of the database. The final stage of archiving consists of saving an archival copy of the waveform data on a mass-store with the appropriate parametric pointers to the data files in the database. 


\section{Data Sources}

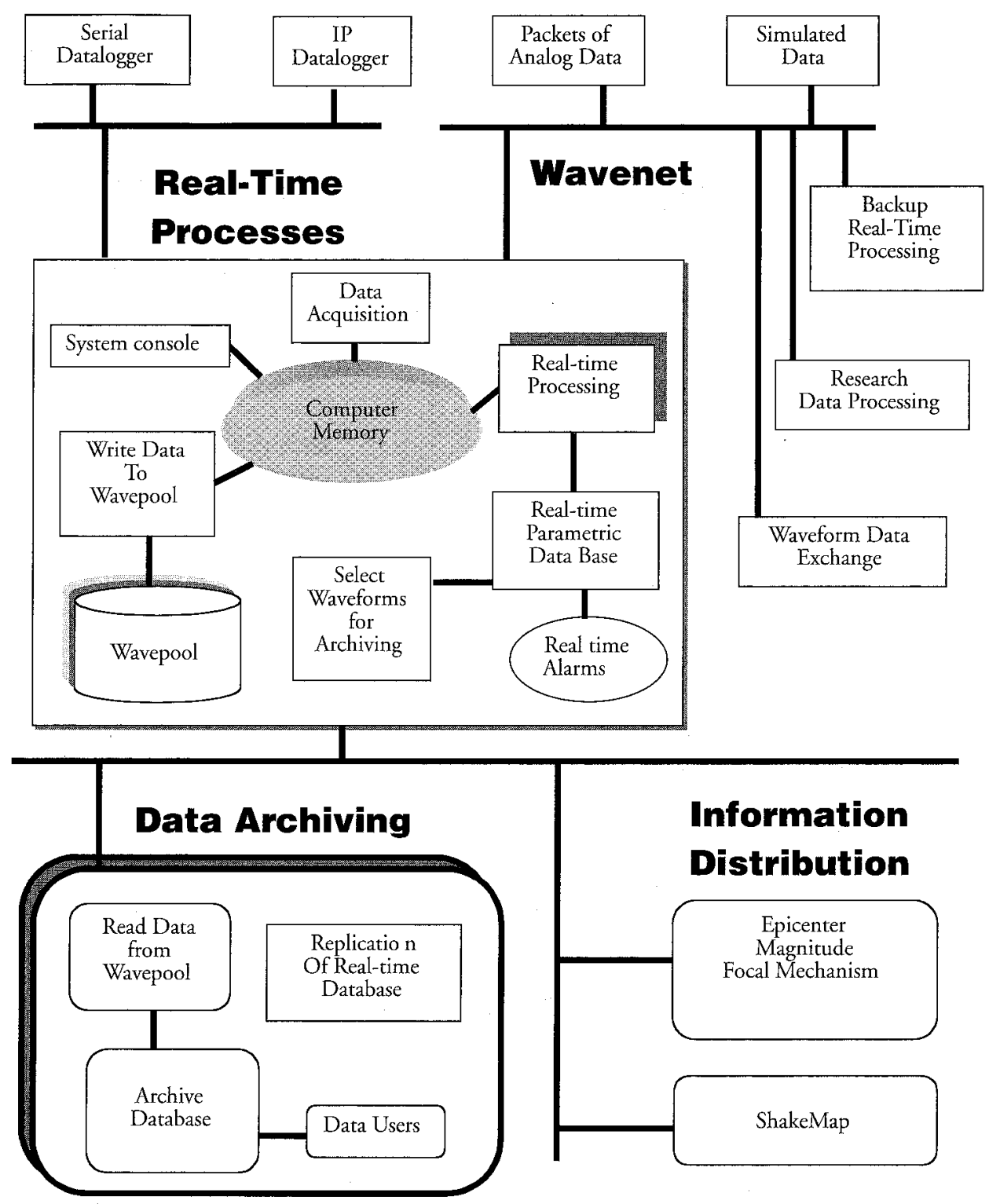

$\Delta$ Figure 5. A schematic flow chart showing the major components of the TriNet real-time software system.

\section{Real-time Data Flow}

Routers, terminal servers, and serial ports are used as physical devices to receive the data. Stations with UDP/IP Ethernet or SLIP-framed UDP/IP communications transmit their data to the main router. The less sophisticated stations use asynchronous serial telemetry to transmit their data to terminal servers. Two Sun Enterprise servers, with eight CPU's each, are operated in a primary and secondary configuration. Should the primary server fail, the secondary server takes the role of being primary.

The main process, comserv, is a public domain software package supported by users and Quanterra, Inc. Comserv acknowledges packets sent by a data telemetry process on the datalogger (whether serial, TCP, or UDP). If a packet does not arrive, comserv does not acknowledge and the datalogger retransmits the packet. Comserv has many clients that access data held in a shared memory area; most are related to realtime processing and network monitoring functions. One comserv client, datalog, populates a disk-based data storage area, called a WavePool, with seven days of time series and state of health logs. This form of data acquisition differs from most other regional seismic networks because it is focused on handling packetized digital data as opposed to streams of serial data (Figure 5). Data imported from other network Earthworm hubs also are directed into a comserv process, providing a uniform interface for data processes downstream.

An important feature of the real-time system is the WaveNet, which provides real-time access to the time-series 
data (Figure 5). Data are broadcast onto the WaveNet, which is a $100 \mathrm{Mbps}$ private local area network. Any computer connected to the WaveNet can acquire the complete waveform data stream and process the real-time waveform data. Currently we use a computer attached to the WaveNet for development of algorithms for prototype early warning.

The three domains shown in Figure 5 are intentionally decoupled. The real-time parametric database and the wavepool act as clutches between the domains. The information distribution or data archive can stall without affecting the real-time systems. Similarly, a calamity in the real-time system will not adversely affect the data archiving or information distribution because they wait for event notification from the real-time system. Both the primary and backup wavepools and the two real-time parametric databases are active simultaneously. The data archive and information distribution systems are designed to know which one is presently working.

\section{WavePool and WaveServer}

The WavePool is populated with time-series data from the short-period stations of the Southern California Seismic Network, broadband and strong-motion TriNet stations, and stations of other regional networks such as the Anza network (Vernon, 1989). The WavePool and WaveServer are software packages that were developed by Small (1999). They store data on disk and can provide rapid dissemination of subsets of the time series over a network without interrupting the creation of the archive. Time series of one week duration for each station are stored in files of one-hour length for each channel on a $400 \mathrm{~Gb}$ RAID disk system. This makes all of the TriNet waveform data accessible through network data requests to local computers or to computers on the Internet. To ensure reliable operation of the data acquisition system, a separate WavePool is operated in parallel to the primary system using a second $400 \mathrm{~Gb}$ RAID disk system on a secondary system.

The real-time processing system declares an event and writes the associated event parametric data into a database. The database tables are replicated to the data center. Two separate programs operated by the processing center retrieve both continuous (20 sps) and triggered data from the WavePool. The triggered data are requested based on "request cards" that specify the streams and time windows of data to archive for a particular event. The data center stores a permanent archive of the waveforms and derived parametric data.

During times of low earthquake activity, Caltech-USGS TriNet can easily handle the load of data processing. During a major earthquake sequence, the processing load will increase significantly because the data compression is no longer as effective and because more data channels are being analyzed. To ensure the reliable operation of TriNet during a major earthquake sequence, the system has been tested with software that allows us to simulate a major earthquake sequence, such as the $1992 M_{w} 7.3$ Landers sequence. This software, along with the capability of flooding the telemetry paths, has allowed us to make the TriNet system more resilient and to ensure its operation under a full load.

\section{Real-time Time-series Data Exchange}

A TriNet computer that acquires data from the WaveNet is used to distribute and receive waveform data without impacting the TriNet primary data acquisition systems. The ORBto-ORB software is used for time-series data exchange with the Anza and the University of Nevada at Reno seismic networks (Harvey et al., 1998). The ORB-to-ORB software is also used to export data to IRIS/DMC. The Earthworm software is used to export data to Tsunami Warning Centers in Alaska and Hawaii and to exchange data with the USGS in Menlo Park. Time-series data are also exported to the U.S. National Seismic Network in Golden Colorado using USNSN software protocols.

\section{Event Detection}

Currently, the rapid processing done by TriNet consists of phase picking, event association, hypocenter determination, magnitude calculation, and declaration of subnet triggers. A picker is operated on all time-series data to find significant arrivals. The arrivals are associated, and, if the association is successful, an event is declared by the real-time system. The hypocenters are calculated using Hypoinverse 2000 (F. Klein, written communication, 2000). Several of the software modules used for real-time processing, such as the associator and picker for short-period data, have been adapted from the Earthworm software package (Johnson et al., 1995).

In addition to the phase-pick associator, events are also declared by an application called Event Detect. This uses the simultaneous recording of significant accelerations at several TriNet stations as a way of declaring an event. This method, intended to be complementary to the phase-pick associator, ensures that the Caltech-USGS TriNet declares an event even if the phase-pick associator cannot locate it.

To facilitate real-time monitoring of earthquake ground motions, Kanamori et al. (1999) developed a continuous monitoring approach. The main advantage of using this approach is the nearly instantaneous continuous availability of the amplitude values of ground-motion acceleration, velocity, displacement (high-passed), Wood-Anderson response, and the narrow-band responses at $0.3,1$, and $3 \mathrm{~s}$ (to be used for determination of response spectra), all with a constant load on the processing system. The continuous WoodAnderson amplitude and the velocity amplitude are used for rapid determination of $M_{L}$ and $M_{E}$.

\section{Network Data Quality Checking}

To monitor the overall network performance and data quality, record sections are routinely produced for large teleseismic and local events. The teleseismic data are also used to generate station delay times and cross-correlation coefficients for a variety of phase arrivals, which can be used for time and waveform quality control. The results of an evaluation of these record sections are provided to the TriNet station main- 
tenance group to facilitate timely repairs of potentially malfunctioning instruments.

As an example, we show in Figure 6 a selection of vertical component waveform data across TriNet for the 11 July 2000 $M_{w} 6.5$ Kodiak Islands event at a depth of $50 \mathrm{~km}$. These displacement waveforms show the direct $P$ and depth phases $(p P$ and $s P)$. The data have been cross-correlated using a $P$-arrival window of about $40 \mathrm{sec}$ and have been lined up relative to station PAS. The dashed trace shows the waveform stacked for the entire network aligned on the $P$-wave arrival, used for comparison with the data recorded at the separate stations (solid trace). To the left of each trace the station name and cross-correlation coefficient with respect to PAS are given. This cross-correlation coefficient gives a measure of the similarity between the station waveform and that recorded at the reference station PAS, with 1.00 being a perfect match. Station DAN is clearly malfunctioning, and there is a high level of long-period noise at stations STG and SCI, reflected by the low cross-correlation coefficients. The low value for station MSJ is due to a strong high-frequency site response, as can be seen directly from a waveform comparison.

\section{RAPID NOTIFICATION}

TriNet is designed to improve emergency response after the next major earthquake in southern California by providing rapid parametric and ShakeMap information for quick response. In addition, the development of new capabilities for improved response, such as prototype early warning, are continuing.

\section{Hypocenter and Magnitude}

TriNet provides rapid notification via paging, e-mail, and World Wide Web pages. Several specialized notifications are also done. The rapid notification is automatic and is only checked by a duty person once that person has received the notification. The manual check usually takes less than 15 minutes to be completed and for a verification to be issued. All of the parameters that are generated as part of the rapid notification process are stored in the Oracle database.

\section{ShakeMap}

TriNet produces "ShakeMap", which shows the distribution of ground shaking for earthquakes of $M \geq 3.5$. ShakeMaps are generated within 3-5 minutes from the instrumental data combined with newly developed relationships between recorded ground-motion parameters and expected shaking intensity values (Wald et al., 1999a). Production of maps is automatic, triggered by any significant earthquake in southern California (see Wald et al., 1999b for more details).

Once an event is declared by the TriNet real-time system, peak amplitudes are determined within a time window, starting at the origin time and with a duration of $90 \mathrm{sec}$, using the Kanamori et al. (1999) continuous monitoring of ground motion. These peak amplitude values are stored in the database and are provided as input to the ShakeMap. In addition,
ShakeMap uses information about the local geology for interpolation between stations.

ShakeMap data files are pushed, using FTP protocol over the Internet or dedicated communications lines, to clients such as utilities that use in-house geographical information systems (GIS) to display the ground shaking information as an overlay on their infrastructure.

TriNet has provided funds to improve loss estimation tools used by the California Office of Emergency Services (OES) to include ShakeMap as input data. TriNet has made ShakeMap available in formats suitable for input into loss estimation tools used by the Federal Emergency Management Agency (FEMA), the California Office of Emergency Services (OES), Los Angeles County, and others. Having loss estimates constrained by observed ground motions as input can greatly improve the accuracy of the loss estimates over empirically based approaches.

Although ShakeMap data files were used for rapid loss estimation calculations for the Hector Mine earthquake, the earthquake had no significant societal impact because the event occurred in an uninhabited region in the southern Mojave Desert. Farther from the epicenter, it caused only moderate shaking in sparsely populated regions of the Mojave Desert and light shaking in urban areas of southern California.

\section{Testing of Early Warning}

Future developments of Caltech-USGS TriNet will include a prototype early warning system. The idea is to "radio ahead or transmit over the Internet" a warning to other parts of the region that strong ground motion is measured in another part of the network area and seismic waves may arrive shortly. Continuous high-speed communications and fast processing afford this opportunity to get the message out quickly, possibly within 10 to 15 seconds of the initiation of a major earthquake. The warning may include the origin of the area of strong shaking, an approximate magnitude estimate, and continued updates until the completion of the rupture. Early warnings may provide the opportunity to take life safety measures and to mitigate hazards, though in a very rapid time frame. Such measures are more suited for automated processes than effective personal safety response.

The seismological research aspects of early warning include developing sophisticated algorithms. These algorithms must quickly recognize if an earthquake is happening and continually estimate its size, rupture direction, and other source parameters, all while the earthquake is in progress. Alerts of future ground motions at a distant place are a much more challenging aspect of early warning. Such alerts need to include the output from the real-time source estimation algorithms and some probability estimates of future development of the source. In addition, local site effects need to be taken into account.

The earthquake early warning component of the TriNet project also included social science studies that involved the Disaster Research Center (DRC) at the University of Dela- 

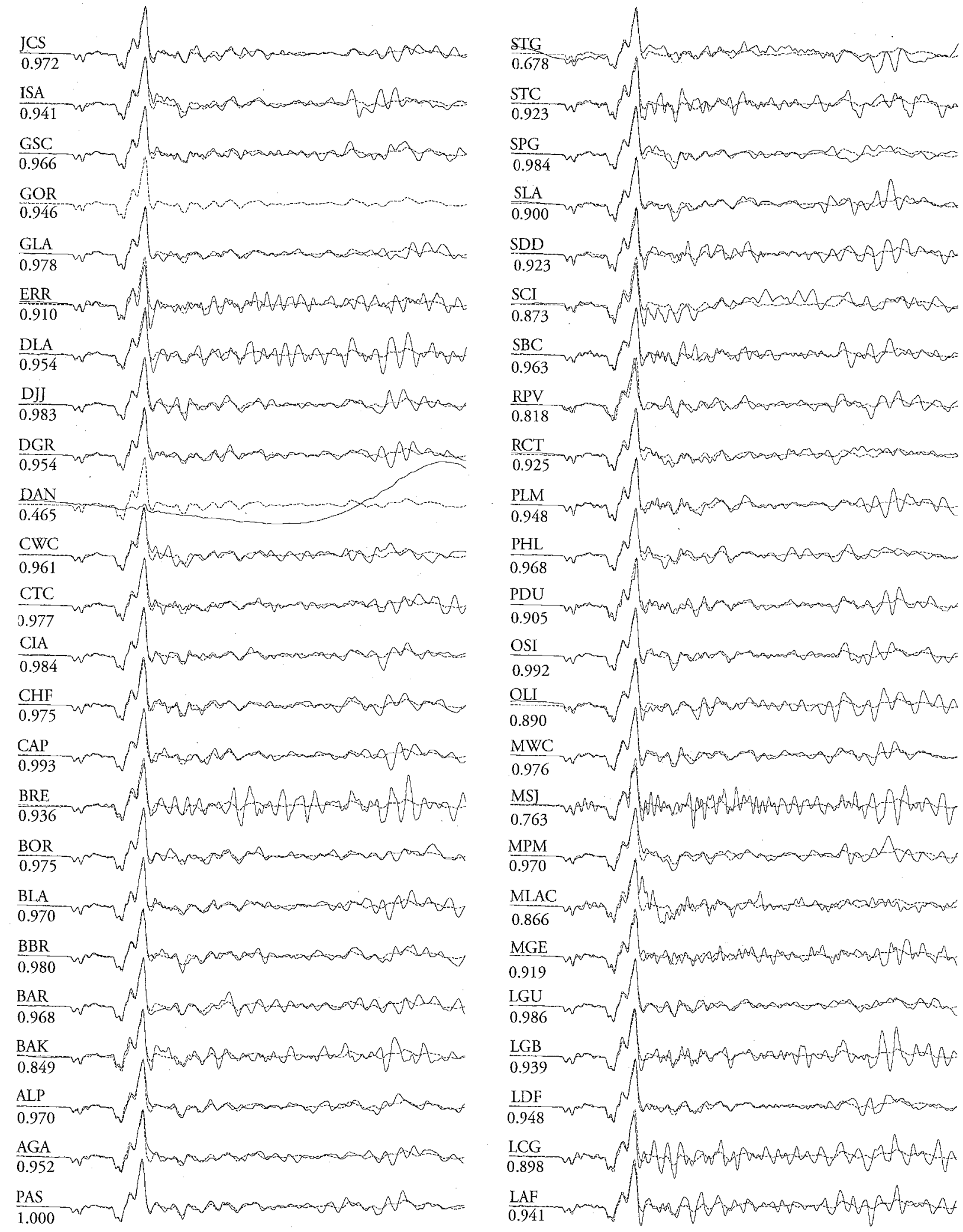

$\begin{array}{llllllllllllll}0 & 10 & 20 & 30 & 40 & 50 & 60 & 70 & 80 & 90 & 100 & 110 & 120 & 130\end{array}$

\section{(seconds)}

$\Delta$ Figure 6. Vertical-component broadband seismograms recorded by TriNet for 11 July 2000 Kodiak Islands; earthquake at $56 \mathrm{~km}$ depth. Waveforms show direct $P$ and depth phases ( $P P$ and $s P$ ). Data have been cross-correlated and lined up with data at station PAS. Dashed trace shows waveform stacked over the entire network for comparison with the data recorded at the separate stations (solid trace). 
ware, the Center for Public Health and Disaster Relief at UCLA, EQE International, Inc., and several TriNet working groups. As a first step, we reviewed the literature in the social sciences for a comprehensive assessment of warning systems for other hazards and behavioral response to warnings. Building on the insights from the social science literature review provided by the DRC, a survey of 200 organizations was conducted by UCLA to assess the acceptability and feasibility of introducing earthquake early warning among four sectors of the community. These sectors included education, health care, emergency management, and utilities and transportation lifelines. With the results of both of these studies in hand, EQE International was asked to address the salient public policy issues raised by the introduction of earthquake early warning in California, including potential legal liabilities, costs and benefits, and the organization and management of a warning system. The objective of these studies is to set the stage for selection of early warning pilot project partners with whom TriNet will work to develop and refine procedures and protocols to test this emerging technology.

\section{POSTPROCESSING, ARCHIVING, AND DISTRIBUTION}

\section{Postprocessing}

The TriNet data flow directly into the database and are available in near real-time to TriNet data analysts and users. The postprocessing that is done by data analysts consists of.modifying parameters in the database. This differs from the old approach of passing the data to the data analysts, who after completing the data analysis would forward the data to the data center.

Jiggle is a graphical earthquake analysis tool developed by TriNet and used by the data analyst to modify the database. Its basic functions are to read or write seismic data from or to any data source in any format. Jiggle displays a user-defined catalog list, waveforms, phase picks, amplitudes, and other parameters. Jiggle allows the manipulation of waveforms for scaling, zooming, filtering, and other functions. It also allows editing and creation of phase picks, amplitudes, codas, and other parameters. Location calculation is done using Hypoinverse 2000 via a client-server implementation. Local magnitude $\left(M_{L}\right)$ calculations are done in the same way as by the TriNet real-time software. Coda amplitude magnitude (MCA) calculation is consistent with the method historically used for the southern California catalog.

Jiggle has been in routine use by TriNet since January 2001. Because it is written in Java, Jiggle is platform-independent and has been run on Solaris, Windows NT v4.0, Windows 98, Windows 2000, and FreeBSD. Access to the seismic data is accomplished by using the Java Abstract Seismic Interface (jasi). It requires the creation of a concrete implementation of the "jasi" class library to access a site-specific data source. The only currently available implementation is to the Northern California Earthquake Data Center (NCEDC) schema used by TriNet.

\section{Near-real-time Archiving and Distribution of Parametric and Waveform Data}

The Southern California Earthquake Data Center (SCEDC) archives and distributes the parametric and waveform data generated by the 375-station Caltech-USGS TriNet. The key element of the design of this integrated system is a common database system, which receives earthquake information as it is being generated by the real-time component of the system, The database and data center operations are discussed in detail by Hafner and Clayton (SRL, this issue).

\section{DISCUSSION}

The ideas behind the design of Caltech-USGS TriNet have evolved from the requirements to both record data and provide rapid notification for the several large magnitude earthquakes such as the $1992 M_{w} 7.3$ Landers, the $1994 M_{w} 6.7$ Northridge, and the $1999 M_{w} 7.1$ Hector Mine earthquakes, They have also been bolstered by new developments in technology. For instance, regional telephone companies made available at no cost frame relay communications from 25 sites for two years. This greatly facilitated the development of inhouse expertise for data acquisition using modern digital packetized communications. Furthermore, the availability of dataloggers with IP interfaces has made it possible to use a variety of communications, including the Internet, for data transmission.

The implementation of Caltech-USGS TriNet is complex, because it is built to be multifunctional and integrates new hardware and software systems. As an example, the requirement of being multifunctional sometimes leads to competing goals between collecting data for seismological research and providing emergency management functionality. The archiving of data that may affect performance of notification functions is an essential part of TriNet, because otherwise the system performance cannot be evaluated in retrospect.

We have completed about $90 \%$ of the development of TriNet. Approximately fifteen stations remain to be installed. Software systems such as the real-time system, the database, and postprocessing are at an advanced stage of development. The prototype early warning software is at an early stage of development.

In October 1999 the $M_{w} 7.1$ Hector Mine earthquake provided a good test and evaluation of the performance of TriNet (Scientists of USGS and SCEC, 2000). At that time about 120 USGS-Caltech real-time stations and about 200 CDMG strong-motion stations, whose data are accessed viaa dial-out protocol, were operational. Within the first minute following the shaking of an event, epicenter, magnitude, and ground-motion parameters are available from the USGS. Caltech element of TriNet, and within minutes ground motion parameters from most of the important near-sourct CDMG stations are reported. A more complete CDMG sta tion report is available within approximately 30 minutes. An initial magnitude and location is obtained within minutes of 
nevent using the real-time stations. For the Hector Mine earthquake, a location and preliminary $M_{L}$ estimate of 6.6 were obtained approximately 90 seconds after the event. An energy magnitude of $M_{E} 7.0$ was obtained approximately 30 seconds later. This parametric information was distributed via paging, e-mail, and the World Wide Web.

The first ShakeMap for the Hector Mine earthquake was produced within four minutes of the event. Initially, ShakeMap ground motions in the near-source region were estimated using ground-motion regression from a point locafion at the epicenter. Later, as information about fault dimensions became available (in the form of aftershock distribution, source rupture models, and observed surface slip), the fault location and dimensions served as the basis for ground-motion estimation in the near-source region.

\section{CONCLUSIONS}

Caltech-USGS TriNet includes new features that have not been used previously by U.S. regional networks. Data from a large number of stations with both broadband and strongmotion sensors will lead to improved understanding of ground motions from both small and large earthquakes. The use of IP communications for almost 200 stations provides new options for the routing of data to several data processing centers. The use of recursive filters on the time-series data is a first attempt at providing real-time event magnitudes at all times and a fairly constant processing load. The reliability of the network is addressed through on-site and central-site data recording, the use of various communication facilities, and multiple processing computers at the central site. Current information about TriNet operations, available software, earthquake information in Southern California, and TriNet seismic data can be found at http://www.trinet.org.

The early planning and implementation of CaltechUSGS TriNet as a program has benefited from the explicit statement of goals. This step in the process provided a backstop for design and implementation decisions encountered along the way, from the choice of seismic hardware and the allocation of technical manpower to the identification of crucial facilities (e.g., air conditioning and power backup refits). This has involved not only decisions at the top management level, but each field technician makes decisions every day, knowing the overall goals are important.

The TriNet project has benefited from the reservoir of experience held by both seismologists and the emergency services community. It is through the cooperative efforts of these diverse disciplines that earthquakes will be better understood and earthquake response will be more rapid and efficient. Based on the success of TriNet, a committee of scientists and emergency managers from northern and southern California is seeking state funding to implement the California Integrated Seismic Network (Oppenheimer et al., 2001). Congtess has authorized and provided initial seed funding for the Advanced National Seismic System (ANSS), a U.S. Geologi- cal Survey project, which would develop a TriNet-like system for the entire nation (Benz et al., 2001).

\section{ACKNOWLEDGMENTS}

We thank the TriNet staff for carrying out the implementation of the TriNet system and our colleagues at CDMG for making the combined CDMG and Caltech-USGS elements of TriNet a reality. Funding for TriNet has been provided by the Federal Emergency Management Agency (FEMA) and the California Governor's Office of Emergency Services (OES) through the Hazards Mitigation Grant Program established following the 1994 Northridge earthquake. The required 25\% cost-sharing is provided by California Institute of Technology, California Trade and Commerce Agency, Caltrans, Ida H. L. Crotty, Verizon California, Donna and Greg Jenkins, Pacific Bell/CalREN, Southern California Edison, Sun Microsystems, Inc., Times Mirror Foundation, and others. Funding has also been provided by the United States Geological Survey from its special 1994 Northridge funds and internal funds, and through contract 01HQAG0007. The Southern California Earthquake Data Center (SCEDC) is also funded by the Southern California Earthquake Center (SCEC). IRIS/GSN provided funds for software support. We also thank Phil Maechling for his contributions to TriNet. P. Friberg and colleagues of ISTI wrote TriNetwatch and provided general software support. Division of Geological and Planetary Sciences contribution number 8811.

\section{REFERENCES}

Benz, H., R. Buland, J. Filson, A. Frankel, and K. Shedlock (2001). The Advanced National Seismic System, Seism. Res. Lett. 72, 70-75.

Gee, L. S., D. S. Neuhauser, D. S. Dreger, M. E. Pasyanos, R. A. Uhrhammer, and B. Romanowicz (1996). Real-time seismology at UC Berkeley: The rapid earthquake data integration project, Bull. Seism. Soc. Am. 86, 936-945.

Hafner, K. and R. Clayton (2001). Seism. Res. Lett. 72, 705-710

Harvey, D. J., D. M. Quinlan, F. L. Vernon, and R. Hansen (1998). ORB: A new real-time data exchange and seismic processing system, Seism. Res. Lett. 69, 165

Hauksson, E., L. M. Jones, and A. Shakal (2002). TriNet, Jennings, P., H. Kanamori, and W. H. K. Lee (editors), Int'l. Handbook of Earthquake and Engineering Seismology: Centennial Publication of the Int. Assn. of Seismology and Physics of the Earth's Interior (in press).

Hutt, R. and H. Bolton (1999). Live seismograms from the Net, IRIS Newsletter 18, 2-4.

Jones, L. M., E. Hauksson, D. Given, R. Clayton, and T. Heaton (1997). System specification of the Caltech/USGS Element of TriNet, unpublished Caltech report.

Johnson, C. E., A. Bittenbinder, B. Bogaert, L. Dietz, and W. Kohler (1995). Earthworm: A flexible approach to seismic network processing, IRIS Newsletter XIV(2), 1-4.

Kanamori, H., E. Hauksson, and T. Heaton (1991). TERRAscope and CUBE Project at Caltech, Eos, Trans. Am. Geophys. U. 72, 564.

Kanamori, H., E. Hauksson, and T. Heaton (1997). Real-time seismology and earthquake hazard mitigation, Nature 390, 461-464.

Kanamori, H., P. Maechling, and E. Hauksson (1999). Continuous monitoring of ground-motion parameters, Bull. Seism. Soc. Am. 89, 311-316. 
Kinoshita, S. (1998). Kyoshin Net (K-NET), Seism. Res. Lett. 69, 309332.

Krawinkler, H., J. C. Anderson, and V. V. Bertero (1995). Steel buildings, in Holmes, W. T., and P. Somers (editors), Northridge Earthquake of January 17, 1994 Reconnaissance Report, Vol. 2: Supplement $C$ to volume 11 of Earthquake Spectra, Earthquake Engineering Research Institute, Oakland, CA, 25-47.

Mori. J., H. Kanamori, J. Davis, E. Hauksson, R. Clayton, T. Heaton, L. Jones, T. Shakal, and R. Porcella (1998). Major improvements in progress for southern California earthquake monitoring. Eos, Trans. Am. Geophys. U. 79, 217-221.

Oppenheimer, D., M. L. Zoback, L. Gee, B. Romanowicz, L. Jones, D. Wald, E. Hauksson, and R. Clayton (2001). The California Integrated Seismic Network, Seism. Res. Lett. 72, 231.

Small, P. (1999). The Caltech/USGS Element of TriNet, Task Description for Waveform archival development build 2, unpublished Caltech report.

Scientists from the U.S. Geological Survey, Southern California Earthquake Center, and California Division of Mines and Geology (2000). Preliminary report on the 16 October 1999 M 7.1 Hector Mine, California, earthquake, Seism. Res. Lett. 71, 11-23.

Teng T.-L., L. Wu, T.-C. Shin, Y.-B. Tsai, and W. H. K. Lee (1997). One minute after: Strong-motion map, effective epicenter, and effective magnitude, Bull. Seism. Soc. Am. 87, 1,209-1,219.

Vernon, F. L. III (1989) Analysis of Data Recorded on the Anza Seismic Network, Ph.D. thesis, University of California at San Diego.

Wald, D. J., V. Quitoriano, T. H. Heaton, and H. Kanamori (1999a). Relationship between peak ground acceleration, peak ground velocity, and Modified Mercalli Intensity for earthquakes in California, Earthq. Spectra 15, 557-564.

Wald, D. J., V. Quitoriano, T. H. Heaton, H. Kanamori, C. W. Scriv ner, and C. B. Worden (1999b). TriNet "ShakeMaps": Rapid generation of peak ground motion and intensity maps for earthquakes in southern California, Earthq. Spectra 15, 537-556.

$$
\begin{array}{r}
\text { California Institute of Technology, } \\
\text { Seismological Laboratory, MC 252-21 } \\
\text { Pasadena, CA 91125 } \\
+1-626-395-6954 \\
\text { hauksson@gps.caltech.edu }
\end{array}
$$

(E.H., P.S., K.H., R.B., R.C., J.G., T.H., K.H., H.K., andJ.P.)

Channel Z Seismometry, Inc., 37 Haynes Avenue Falmouth, MA 02450 (RB.)

U.S. Geological Survey, 525 S. Wilson Avenue Pasadena, CA 91106 (D.G., L.M.J., D.W.) 